\title{
Dose-Dense Therapy
}

\author{
Chair: $\quad$ Michael Untch, Berlin \\ Participants: Jonas Bergh, Stockholm Mark Citron, New York, Clifford A. Hudis, New York \\ Jens Huober, St. Gallen Volker Möbus, Frankfurt Günter Steger, Wien
}

\begin{abstract}
Question 1: What is the role of dose-dense therapy for breast cancer patients in the year 2008? Do you think that dose-dense therapy is one of the possible standards for adjuvant chemotherapy in breast cancer patients?
\end{abstract}

Bergh: As I have understood it dose-dense therapy is not used as standard therapy outside the clinical study setting in Scandinavia. There are of course data available in the scientific community which would be supportive of such a strategy. There should be room for identification of other regimens and therapy concepts making use of the dose-dense strategy.

Citron: Dose-dense chemotherapy is one the leading postoperative treatments for breast cancer, becoming one of the standards for adjuvant therapy in the US. Superior efficacy, reduced toxicity, and patient convenience lead a list of factors.

Hudis: It is definitely a standard. In CALGB 9741 the every other week (dose-dense) treatment was associated with multiple benefits compared to every third week (standard) AC (cyclophosphamide and doxorubicin) followed by paclitaxel (Citron et al., JCO 2003, Hudis et al., SABCS 2005). Firstly, it improved both disease-free and overall survival. Secondly, it was associated with reduced risks of several important toxicities including neutropenic fever and, surprisingly, grade 3 cardiac events. Finally, it shortened the time of treatment. For patients using AC followed by paclitaxel it is harder to justify every third week treatment than the dose-dense regimen.

Huober: There were several studies showing that dose-dense or dose-dense and dose-intensified chemotherapy is an effective and safe adjuvant treatment in high-risk breast cancer patients. In two well designed phase 3 studies by Moebus et al. (ETC trial) and Hudis et al. this approach proved to be superior to conventional chemotherapy with regard to disease-free survival (DFS) and overall survival (OS). Therefore dose- dense chemotherapy can be a valid option for patients with tumors with high-risk features. Still there are several questions unanswered. We do not know the best regimen (shall we only shorten the intervals or additionally increase the dose?), we need to know which patient population will benefit the most from this approach. Moreover, what was not known when these trials have been designed that the comparator $4 \times \mathrm{AC} /$ EC (epirubicin and cyclophosphamide) followed by $4 \times$ paclitaxel every three weeks is not the most effective adjuvant regimen (in recent studies it was inferior to Canadian CEF (cyclophosphamide, epirubicin and fluorouracil) and to $4 \times \mathrm{AC}$ followed by paclitaxel weekly, at least in a hormone receptor negative population). Further studies with more effective comparators will give more clarity.

Möbus: Indeed, dose-dense chemotherapy is one of the possible standards of adjuvant chemotherapy, at least in high-risk breast cancer patients. Two large randomized trials have shown independently from each other (CALGB C9741 and ETC trial of the AGO), that dose-dense chemotherapy ( $q 2 w$ ) leads to a superior DFS and OS in comparison to conventionally dosed chemotherapy $(\mathrm{q} 3 \mathrm{w})$. These are mature data regarding the median follow-up duration of 5 years in both trials.

Steger: In high-risk patients fit for this therapeutic approach dose-dense therapy might be considered and is one of the possible standards based on the published data.

Question 2: Would you also use dose-dense therapy not just for adjuvant but also for neoadjuvant treatment of breast cancer patients?

Bergh: In principle I can see no contraindication from the news in the neoadjuvant setting. However, personally I would like to start with patients with receptor-negative disease and

\begin{tabular}{|c|c|}
\hline KARGER & (C) 2008 S. Karger GmbH, Freiburg \\
\hline $\begin{array}{l}\text { Fax +49 } 7614520714 \\
\text { E-mail Information@Karger.de } \\
\text { www.karger.com }\end{array}$ & $\begin{array}{l}\text { Accessible online at: } \\
\text { www.karger.com/brc }\end{array}$ \\
\hline
\end{tabular}

Prof. Dr. med. Michael Untch

HELIOS Klinikum Berlin-Buch, Frauenklinik

Akademisches LK der Universität Charité

Schwanebecker Chaussee 50,13125 Berlin, Germany

Tel. + 4930 9401-53300, Fax -53309

E-mail michael.untch@helios-kliniken.de 
those with a high proliferation in their tumors. Whether that is correct or not I do not know but it should be tested.

Citron: The objective of neoadjuvant chemotherapy is to downstage tumor size, eliminate microscopic metastases, and facilitate surgical goals. Study C9741 established the superior efficacy of adjuvant dose-dense therapy over conventional treatment. Although not proven in the neoadjuvant setting, emerging results support the adjuvant data. There are scheduling and time frame issues as well. The 4-month regimen of dose-dense $\mathrm{AC} \rightarrow \mathrm{T}$ (tamoxifen), allows the delivery of a full 8 courses over a 4-month period, allowing surgery within a reasonable time period. The conventional every-3-week, 6-month neoadjuvant regimen is often interrupted by surgery and ensuing recovery. This break in the continuity of treatment reduces maximal tumor shrinkage and theoretically may permit tumor regrowth.

Hudis: Absolutely. The goals of pre-operative treatment include both in-breast response and control of micrometastatic disease, and it is the latter that drives overall outcomes. If every third week is inferior to every second week treatment how can one justify compromising disease-free and overall survival to administer treatment at that less effective interval? There is certainly no evidence of an inferior in-breast response to dose-dense therapy and the question has not been asked in a precise fashion but it is the distant metastases that matter in the long-term in any case.

Huober: There are limited data regarding dose-dense therapy as a neoadjuvant approach. There were two trials conducted by Untch et al. showing good reponse rates (pCR 18-22\%) with dose-dense paclitaxel and epirubicin. However, the best evaluated regimen in the neoadjuvant setting is $4 \times \mathrm{AC} / \mathrm{EC}$ followed by $4 \times$ docetaxel with very consistent and robust results in different trials (NSABP-B27, Geparduo and Geparquattro) and a pathological response rate of $26-30 \%$. Thus outside a clinical trial I would prefer this regimen.

Möbus: At the San Antonio Meeting 2007, the results of a phase 3 study comparing conventionally dosed chemotherapy with dose-dense and dose-escalated chemotherapy have been reported. Again, dose-dense and dose-escalated chemotherapy leads not only to an improvement of cCR (clinical complete remission) and pCR (pathological CR), but also to an improvement of DFS and OS. However, we prefer primary surgery followed by adjuvant chemotherapy. The number of positive nodes remains the most important risk factor in breast cancer, which cannot be evaluated after neoadjuvant chemotherapy. Neoadjuvant chemotherapy remains the domain of testing new drugs and concepts.

Steger: For the neoadjuvant approach dose-dense chemotherapy should not be used outside clinical trials as long as neoad- juvant therapy has not shown a definite survival benefit. For downstaging purposes to allow breast conservation the dosedense approach is not necessary.

\section{Question 3: What is the best schedule to be used as dose-dense therapy? Would you use dose-dense therapy in the initial definition by shortening the intervals between chemotherapies from 3 to 2 weeks at the same dose per cycle and the same total dose or would you also combine the dose-dense therapy with an increase of the dose per cycle and therefore an increase of the total dose of chemotherapy?}

Bergh: According to the guidebook same doses as used for the three-weekly strategy should then be tested in the two-weekly strategy although this strategy has to be supported by G-CSF (granulocyte-colony stimulating factor). You can of course also in the next step think to escalate the doses for some of the drugs. In Scandinavia we have tried to perform studies where we take into the account the individual patient sensitivity to the drug. There are ample of retrospective data describing a worse outcome for those patients who have minimal toxicity on conventional chemotherapy. The dose is reduced for those who experience toxicity - the same principle could be applied for those who experience no toxicity: the dose could be escalated in the dose-dense strategy. Just to escalate the dose in all patients is not intellectually sound according to our strategy. Presently we have a multinational setting exploring our strategy to escalate the dose on an individual basis.

Citron: Study C9741 presented two separate dose-dense schedules, a 4-month regimen combined 2 drugs (AC) followed by a single agent (T), a 6-month plan employed sequential single agents $(\mathrm{A} \rightarrow \mathrm{T} \rightarrow \mathrm{C})$. Both had the same agents, doses and cumulative drug doses. Only the duration differed. The 6-month schedule is preferred in older patients or those with co-morbidities because of reduced emesis, anemia, and fatigue. If tolerated, the 4-month schedule is preferred. The optimal drug dose should always be administered. Dose escalation should only be implemented if there is demonstrated superiority to standard dose. There is no supporting evidence for increasing the dose of drugs used in C9741.

Hudis: There are studies addressing each of these issues. Simple dose escalation of doxorubicin, cyclophosphamide, and paclitaxel was not effective in NSABP and CALGB trials. Strict testing of dose-density (controlling for cycle number and dose size) demonstrated that schedule - in isolation - is important. The German trials of dose-density demonstrate advantages as well while incorporating both dose-dense scheduling and dose-escalation. The only way to determine if both manoeuvres are needed would be to conduct a randomized trial on this question. 
Huober: We have two positive trials with either approach. In both trials the dose-dense or dose-dense and dose-intensified regimen was superior to the classical $4 \times \mathrm{AC} / \mathrm{EC}$ followed by $4 \times$ paclitaxel. Even though dose intensification of the single drugs epirubicin, paclitaxel and cyclophospamide did not show superiority over conventional dose in some other trials we can not exclude a contribution of dose intensification in addition to dose densitiy in the ETC trial to the favorable outcome in this high-risk population. Since we have no direct comparison of these regimens it is not possible to prefer one of these regimens based on current data. As I contributed several patients to the ETC trial and gained experience with this regimen for practical reasons I would use this approach.

Möbus: We have learned a lot about dose-dense chemotherapy in the last 4 years. Most important: the concept of dose density does not automatically lead to an improvement in comparison with conventionally scheduled chemotherapy. We have negative studies in the adjuvant and in the neoadjuvant setting (GONOMIG, GEPARDUO), but these negative results can be explained clearly. When the total dose of one important drug (epirubicin) is to low (GONO-MIG; total dose of epirubicin only $360 \mathrm{mg} / \mathrm{m}^{2}$ ) or the duration of treatment is to short (GEPARDUO: only 4 cycles of dose-dense doxorubicin/docetaxel treatment compared to 8 cycles of standard chemotherapy), the concept of dose density fails. Considering carefully the contribution of dose density and dose escalation in optimizing tumor cell kill, I also believe that the concept of dose density is more important than the concept of dose escalation. However, dose escalation may also play a role in addition to dose density. The ETC trial, which was dose-dense and dose-escalated, has shown the best results that have ever been reported in a highrisk population with a median of 8 positive lymph nodes (5-year OS of $82 \%$ !). Therefore, when treating high-risk patients outside of a clinical trial, we favor the dose-dense and dose-escalated ETC regimen above the dose-dense ATC regimen.

Steger: For the time being dose-dense therapy should be used at the same dose per cycle every 2 weeks and at the same total dose. Further individualization of the dose based on the individual (hematological) side effects to increase the total dose or to avoid unnecessary toxicity in the adjuvant setting might be of value and one such trial is currently ongoing in Sweden and in Austria with the Germans soon to join (SBG 2004-1/ ABCSG 25). The results of such trials should be awaited.

\section{Question 4: For which patients do you think is dose- dense therapy most appropriate? Do you use dose-dense therapy just in axillary lymph node-positive patients?}

Bergh: Patients with lymph node-positive disease should be candidates for such a study. In the future it may be that we first should identify responsiveness to chemotherapy and that will then be combined with selection of a certain risk level. The potential benefit versus potential risk would serve as guidance.

Citron: The majority of node-positive and certain subsets of node-negative patients are candidates for dose-dense chemotherapy. Study C9741 established the benefit of dose-dense treatment in lymph node-positive patients. It is commonly accepted to gauge risk by nodal status, but this arbitrary distinction does not factor in the basic biology of aggressive nodenegative disease. This distinction begins with triple-negative, node-negative patients with tumors greater than $1.5 \mathrm{~cm}$. The benefit of dose-dense therapy should be weighed against competing concerns of toxicity, risk of myelodysplasdic syndrome / acute myelogenous leukemia (MDS/AML), and cardiac toxicity. Dose-dense is generally not called for in ER-positive, HER2-negative, node-negative patients, with micrometastses in one node, and may not be optimal for 1-2-node patients over 60 with co-morbidities. Here a non-anthracycline q 3 week regimen is generally indicated. For ER-positive, HER2negative patients with a minimum of 3 positive nodes, dosedense treatment is appropriate. It is the 1-2 node 'gray area' that is the toughest call deciding the benefits versus risks of such intensive therapy. Pivotal factors include age, co-morbidities, and pathological characteristics amongst others.

Hudis: Any patient who is deemed a candidate (by virtue of positive nodes or perhaps a 'high-risk' node-negative subtype) for an $\mathrm{AC}$ and taxane regimen is a candidate for dose-dense therapy.

Huober: One of the main messages from the recent consensus conference in St. Gallen regarding adjuvant treatment decisions in breast cancer was first to consider treatment responsiveness and then to consider the risk. Several studies suggest that that hormone receptor-negative tumors or hormone receptor-positive tumors that are HER2-positive or the triple negative tumors are far more sensitive to chemotherapy than the others. Based on these data it is not only the number of positive axillary nodes but also these factors guiding our adjuvant treatment decisions. Therefore it might be appropriate to consider a younger patient with hormone receptor-negative, poorly differentiated disease with only a few positive lymph nodes for intense chemotherapy.

Möbus: Outside of a clinical trial we are giving dose-dense and dose-escalated ETC to all high-risk patients with 4 and more positive nodes and younger than 65 years of age. In patients with 1-3 positive nodes, this recommendation depends on the presence of additional risk factors (e.g. young age, grading III, hormone receptor negative or only weakly positive).

Steger: If treated outside a clinical trial the high-risk definition of the St. Gallen consensus is used to consider dose-dense 
therapy. Triple-negativity of the tumor together with other risk factors like young age might also be a trigger to think of this option.

\section{Question 5: Which drugs do you use to avoid side effects? Do you routinely use leukocyte and erythrocyte supporting bone marrow factors and/or antibiotic prophylaxis?}

Bergh: With present knowledge of uncertainty regarding the potential negative effect with erytropoietins I would strongly be against the use of this group of drugs in this setting where we have a curative intention. A most recent metaanalysis demonstrates a $10 \%$ negative effect on overall survival by the use of erytropoietin, although as expected, with marked heterogeneity (Bennet et al., JAMA 2008). I have serious difficulties to see that there are safe levels for hemoglobin as some investigators have claimed. If the negative effect is due to the expression of receptors on breast cancer cells a safe level for hemoglobin is very difficult to understand. Regarding Granulocyte-colony stimulating factor (G-CSF) I do not think that the data of concern are as strong for G-CSFs although I personally have concerns to give it directly after chemotherapy while G-CSF may stimulate marrow-cells which may have been damaged by the chemotherapy. This is not hard evidence but we do have retrospective data supporting that the delayed delivery of GCSF combined with lower accumulative doses of epirubicin and cyclophosphamide seems to be beneficial in the reduction of the risk of secondary MDS/AML. From an ecological point of view the use of antibiotics should be minimal, but they are likely required for at least some dose-dense strategies and some of the used drugs. We are presently in our multinational ongoing study with dose-dense docetaxel not using it during this part of the study but during the EC-part.

Citron: Conventional treatment to prevent neutropenia after combined AC in dose-dense therapy, includes filgrastim and pegfilgrastim. During the single-agent paclitaxel phase in both the 4- and 6-month dose-dense schedules, the number of doses of filgrastim can be attenuated or eliminated without myelosuppressive effects. This is also true for the single-agent cyclophosphamide phase of the 6-month $\mathrm{A} \rightarrow \mathrm{T} \rightarrow \mathrm{C}$ dosedense regimen. The routine use of antiobiotic prophylaxis is mitigated due to the low incidence of infection in C9741. Redcell growth factors follow ASCO guidelines.

Hudis: All patients by definition require G-CSP. While the pilot trials and C9741 used short-acting filgrastim, non-randomized trials have incorporated pegylated G-CSF with no evidence of a loss of safety. Hence we routinely use the longacting form. In terms of ESA (erythropoietic stimulating agents), C9741 documented an increased risk of anemia and transfusion requirement and subsequent trials suggested that these agents could abrogate this issue. However, lingering concerns regarding the acute safety of ESA in cured patients has led to a conservative role for these agents in the adjuvant setting.

Huober: For safe application and to guarantee the tight intervals G-CSF are mandatory for dose-dense and dose-intensified therapy. In some regimens also the additional prophylactic use of antibiotics can be necessary to decrease the rate of febrile neutropenias. It is proven that the use of ESA can reduce blood transfusions and maintain the hemoglobin during chemotherapy. However the safety of ESA is under debate since a recently published meta-analysis showed an increased mortality risk and increased risk of thrombembolic events with these drugs. Thus until the safety concerns have not been cleared I would be very careful and restrictive outside a clinical trial with the use of ESA even in dose-dense or intensified regimens.

Möbus: We routinely give antiemetic treatment. For all dosedense regimens G-CSF or pegfilgrastim support is mandatory. We also know that nearly $25 \%$ of the patients need red blood cell transfusions, when the ETC regimen is given without ESA. In 2007, we have reported the 5-year results comparing ETC with or without epoetin-alpha. There was no difference in 5-year DFS and OS between the two arms, but the number of transfusions was highly significant diminished in the ETC arm with epoetin alpha. We conclude, that dose-dense chemotherapy in combination with ESA is safe, avoids transfusions and enables $\mathrm{Hb}$ values in the normal range.

Steger: We use G-CSF or pegylated G-CSF routinely and prescribe antibiotic prophylaxis only as a secondary preventive measure. We try to avoid ESA in the adjuvant setting not only because they have come into discussion lately for possibly negatively influencing cancer prognosis, but because the time to response after development of significant anemia is too long and clinically most often not useful. Instead we recommend packed erythrocyte transfusion if relevant and/or symptomatic anemia develops.

\section{Question 6: What is the percentage of patients in your hospital/country treated with dose-dense therapy on a routine basis outside of clinical trials?}

Bergh: I can not give you the exact percentage but we inform all patients who fulfill inclusion criteria about our ongoing study. Half of those who accept participation will be randomized to dose-dense therapy. Presently we have a high acceptance rate for participation in the study.

Citron: Almost two-thirds of America's oncologists and investigators now prefer dose-dense $\mathrm{AC} \rightarrow \mathrm{T}$ therapy when treat- 
ing node-positive, HER2-negative tumors, according to 2007's 'Patterns of Care' survey by Dr. Neil Love.

Hudis: I am not sure but it is large.

Huober: Currently we do not use dose dense-regimens outside clinical trials.

Möbus: Up to now we are treating all high-risk patients in a clinical trial (GAIN trial). In our hospital, approximately $50-60 \%$ of all node-positive patients receive dose-dense chemotherapy.

Steger: Since we currently do have an appropriate protocol, we try to treat every patient within SBG 2004-1/ABCSG 25.

\section{Question 7: Do you use dose-dense therapy with trastuzumab (or trastuzumab after dose-dense} therapy)? If not, would you be interested in participating in a trial with trastuzumab and dose-dense therapy?

Bergh: We are using trastuzumab in sequence after chemotherapy as done in the HERA-study. Although in our present study you are also allowed to use trastuzumab together with dose-dense therapy.

Citron: Based on risk and co-morbidities, dose-dense therapy with trastuzumab is a leading option.

Hudis: Yes. We have reported on the cardiac safety of this approach (Dang et al., JCO 2008) and have been using this regimen off-study for several years.

Huober: We have only limited data about the safety of dosedense therapy in combination with trastuzumab (Dang et al., JCO 2008) and no data about the use with other HER2-directed agents like lapatinib so that outside a clinical trial I would use trastuzumab only after completion of chemotherapy. Further, we do not know at this time if the concomitant application of chemotherapy and trastuzumab in the adjuvant setting is superior to sequential therapy. The mature results of the NCCTG N9831 trial regarding this issue are pending. Moreover the optimal use of the anti-HER2 therapy is probably more important and of higher research priority than the investigation of different chemotherapy schedules in this patient population.

Möbus: At our department, trastuzumab is given after dosedense chemotherapy. A trial of simultaneous dose-dense chemotherapy and trastuzumab would close the gap of knowledge in this field.

Steger: No, such a trial would be very interesting.

\section{Participants}

Professor Jonas Bergh, M.D., PhD

Scientific Director Karolinska Oncology

Radiumhemmet

Karolinska Institutet and University Hospital, Solna

17176 Stockholm, Sweden

E-Mail jonas.bergh@ki.se

Mark L. Citron, M.D.

Albert Einstein College of Medicine 2800 Marcus Avenue

Lake Success, NY 11042, USA

E-Mailmcitron@aol.com

Clifford A. Hudis, M.D.

Memorial Sloan-Kettering Cancer Center

Division of Solid Tumor Oncology

1275 York Ave., NY 10021, USA

E-Mail hudisc@mskcc.org

Prof. Dr. med. Jens Huober

Kantonsspital St. Gallen

9007 St. Gallen, Switzerland

E-Mail Jens.huober@kssg.ch

Prof. Dr. med. V. Möbus

Frauenklinik

Städtische Kliniken Ffm-Höchst

Postfach 800769

65907 Frankfurt a. M., Germany

MoebusVolk@aol.com

Univ.-Prof. Dr. med. Günther Steger

Medizinische Universität Wien

Klinische Abteilung für Onkologie

Währinger Gürtel 18-20

1090 Wien, Austria

E-mail guenther.steger@meduniwien.ac.at 